\title{
Assessing the Archaeoparasitological Potential of Quids As a Source Material for Immunodiagnostic Analyses
}

\author{
Johnica J. Morrow*, Karl J. Reinhard \\ Pathoecology Laboratory, School of Natural Resources, University of Nebraska-Lincoln, 3310 Holdrege Street, Lincoln, Nebraska 68583-0962, USA
}

\begin{abstract}
In the present study, quids from La Cueva de los Muertos Chiquitos (CMC) were subjected to ELISA tests for 2 protozoan parasites, Toxoplasma gondii $(n=45)$ and Trypanosoma cruzi $(n=43)$. The people who occupied CMC, the Loma San Gabriel, lived throughout much of present-day Durango and Zacatecas in Mexico. The known pathoecology of these people puts them into at-risk categories for the transmission of $T$. gondii and T. cruzi. Human antibodies created in response to these 2 parasites can be detected in modern saliva using ELISA kits intended for use with human serum. For these reasons, quids were reconstituted and subjected to ELISA testing. All test wells yielded negative results. These results could be a factor of improper methods because there is no precedence for this work in the existing literature. The results could equally be a simple matter of parasite absence among those people who occupied CMC. A final consideration is the taphonomy of human antibodies and whether or not ELISA is a sufficient method for recovering antibodies from archaeological contexts. An additional ELISA test targeting secretory $\lg A$ (slgA) was conducted to further examine the failure to detect parasite-induced antibodies from quids. Herein, the methods used for quid preparation and ELISA procedures are described so that they can be further developed by future researchers. The results are discussed in light of the potential future of quid analysis.
\end{abstract}

Key words: Toxoplasma gondii, Trypanosoma cruzi, archaeoparasitology, archaeoserology, ELISA, La Cueva de los Muertos Chiquitos, quid

\section{INTRODUCTION}

Employing ELISAs as a means of examining archaeological materials for evidence of protozoan parasites has become increasingly more routine among archaeoparasitological researchers [1-15]. Much of the existing work has focused on the recovery of protozoan parasite antigens from latrine sediments [4,9-11,13,14]. In addition to sediments, Gonçalves et al. [4] examined 22 coprolites from Brazil, Chile, and Sudan using an ELISA kit for detecting Entamoeba histolytica coproantigens and did not find any positive samples. Allison et al. [1] used ELISAs to test coprolites from the intestinal tracts of mummies for Cryptosporidium sp. and Giardia sp. antigens. Another study tested coprolites for Giardia duodenalis antigens and found 4/84 to be positive [2]. Recently, Morrow and Reinhard [15] recovered Cryptosporidium parvum coproantigens from 66/90

\footnotetext{
- Received 2 May 2016, revised 21 June 2016, accepted 4 July 2016.

*Corresponding author (johnica@huskers.unl.edu)

(c) 2016, Korean Society for Parasitology and Tropical Medicine

This is an Open Access article distributed under the terms of the Creative Commons

Attribution Non-Commercial License (http://creativecommons.org/licenses/by-nc/4.0)

which permits unrestricted non-commercial use, distribution, and reproduction in any

medium, provided the original work is properly cited.
}

coprolites excavated from a cave in Durango, Mexico.

Quids, which are expectorated masses of human-masticated plant fibers, are desiccated artifacts commonly found at New World archaeological sites. These artifacts are frequently overlooked despite their potential importance in the interpretation of ancient diets and diseases. Quid analyses have involved plant fiber identification, dental impressions, and phytolith recovery [16-19]. Such studies have examined the role of quid chewing in the development of dental wear among archaeological populations. Hammerl and colleagues [19] were also able to use dental impressions of quids to recover demographic (age) data. The quids were all phytolith-rich and most were derived from Agave plant fibers, although maize leaves and husks were also identified among some of the quids [19]. The phytoliths of Agave are quite abrasive, capable of inflicting damage to substances as resilient as tooth enamel [18]. Because these phytoliths, along with those of other dietary abrasives recovered from coprolites and quids $[18,20]$, are able to cause dental wear, it is likely that they may have also created microlacerations within the soft tissues of the mouth. Such microlacerations would have caused minor bleeding, releasing a flood of biomolecules into the mouth as a quid bolus was 
formed. This creates the potential for such biomolecules to become integrated within the quid bolus prior to expectoration. Salivary antibodies, such as secretory IgA (sIgA), would have become similarly integrated into the quid bolus.

A few studies have employed ELISA tests for the purposes of diagnosing parasitic infections using modern saliva $[22,23]$. The recovery of species-specific parasite-induced antibodies from human saliva in modern contexts begs the question: Can these antibodies be recovered from archaeological materials saturated with desiccated human saliva? To answer this question, one first must contemplate the preservation potential of such antibodies. While parasite-specific coproantigens have been demonstrated to preserve within archaeological materials like coprolites and latrine sediments, the recovery of humancreated parasite-specific antibodies from archaeological materials has not been attempted to date. Thus, the preservation and degradation of antibodies from an archaeological perspective have not been fully explored. However, researchers working with modern samples have reported long-term stability of salivary biomolecules $[24,25]$. Therefore, the potential persistence of parasite-induced human immunoglobulins is conceptually plausible. An ideal archaeological potential source material for salivary immunoglobulins is the often-ignored quid. With the capability to incorporate antibodies from the saliva as well as from blood released via microlacerations in the oral cavity caused by phytoliths, these artifacts provide a mechanism for assessing archaeological parasitism in a unique way. To date, no previous studies have employed ELISA techniques for examining quids. The present study represents the first effort to analyze quids $(n=45)$ for the presence of 2 species of protozoan parasites (Toxoplasma gondii and Trypanosoma cruzi).

The quids used in the present study were excavated from La Cueva de los Muertos Chiquitos (CMC), a site within the Rio Zape Valley, in the early 1960s. This valley lies approximately $18 \mathrm{~km}$ southeast of Guanaceví in Durango, Mexico, and is home to a number of caves utilized by the Loma San Gabriel. The region illustrates a cultural transition zone between the northern most edge of Mesoamerica and the greater American Southwest [26-28]. CMC was used year-round as a temporary habitation by the Loma San Gabriel between 1,200 and 1,400 years ago $[29,30]$. This cave housed an abundance of botanical artifacts, the skeletons of 14 children (aged several months to 5 years at the time of death) as well as some adult bone fragments, nearly 500 coprolites, and over 2,000 quids all sealed beneath adobe floors $[28,29,31,32]$. The people who utilized
CMC subsisted using a mixed strategy of agricultural production and hunting-gathering, both of which fluctuated seasonally $[29,33]$. Previous analyses of coprolites reported excellent preservation of parasitic helminth eggs [34], bacterial DNA [35], and parasite coproantigens [15]. The incredible preservation of both physical and molecular parasite evidence makes this site ideal for testing new methods of parasite evidence recovery utilizing quids as source materials.

Previous studies of material from CMC have shown that those utilizing the cave had living and nutritional behavior patterns that likely perpetuated the life cycles of certain parasites $[15,33,36]$. As these people modified their environments to survive, they may have inadvertently promoted the transmission of many different types of parasites, including T. cruzi and T. gondii. Evidence of T. cruzi has been recovered from archaeological materials in North and South America [37-44]. The sylvatic cycle of $T$. cruzi involves a triatomine bug that serves as a vector for the parasite and a mammalian definitive host. About 180 species of mammals, including bats, carnivores, rodents, ungulates, and primates, have been identified as reservoir hosts for T. cruzi. Domestic cycles of T. cruzi are perpetuated in human populations via vectored transmission and via oral transmission of contaminated foods [45].

From an archaeological perspective, this parasite is particularly interesting because prehistoric humans of the Southwestern USA and Mesoamerica inserted themselves into the T. cruzi life cycle as they changed their environments to better suit their survival needs. Reinhard and Araújo [44] discuss the phenomenon of anthropogenic changes to natural habitats that led to an increase in vector populations. Simultaneously, humans induced population decline in reservoir hosts via woodrat hunting and habitat displacement, which caused the vectors to come in contact with human hosts more frequently than in the past [46]. These behaviors placed humans at risk for contracting zoonotic trypanosomiasis that eventually became the American trypanosomiasis (Chagas' disease) that infects an estimated 10 million people today [47]. The origins of T. cruzi can be traced back more than 9,000 years in the New World. Analyses of material from mummies place the establishment of this parasite in the Americas long before European contact [39,41,48-50]. Guhl and colleagues [51] explored the links between Chagas disease and human migration.

Artifacts from CMC have not previously been tested for evidence of T. cruzi; however, dietary evidence of small rodent consumption at this site supports the hypothesis that this par- 
asite may have been cycling among the Loma San Gabriel at CMC in prehistory. The majority of evidence regarding T. cruzi from archaeological contexts has been collected using molecular techniques that involve the recovery of DNA [52,53]. One study demonstrated that T. cruzi DNA could be recovered from experimentally desiccated mouse tissues [54]. To date, ELISA techniques have not been applied to archaeological materials to recover evidence of T. cruzi. Because commercial ELISA kits for T. cruzi are designed to test human serum rather than fecal samples, there has not been a practical way of utilizing this technique in the search for Chagas' disease in prehistory. Pinho and colleagues [23] demonstrated that T. cruzi antibodies could be recovered from modern saliva using ELISA kits. If these antibodies survive following desiccation, there is the potential that ELISA kits could prove to be useful for the recovery of T. cruzi evidence from saliva-contaminated artifacts, such as the quids excavated from CMC. If the use of ELISA techniques in this regard is successful, the archaeoparasitological community could enjoy a new rapid, reliable, and cost-effective method for recovering evidence of $T$. cruzi from archaeological quids.

The origins of toxoplasmosis among humans have been more elusive than those of American trypanosomiasis. The genus Toxoplasma is approximately 10 million years old, with Toxoplasma gondii originating sometime in the last 10,000 years [55]. Today, about a third of the global human population is infected with this parasite [56]. In the United States, this parasite is responsible for over 300 deaths and 4,000 hospitalizations annually. In fact, it has been deemed the second most important foodborne pathogen in the nation [57]. Within the state of Durango, Mexico, modern rural populations have approximately $35.8 \%$ seroprevalence for $T$. gondii [58]. AlvaradoEsquivel and colleagues [59] conducted a modern seroepidemiological study and reported 30.3\% seropositivity within a Mennonite community in a rural region of Durango, Mexico, as compared to $6.1-12 \%$ seropositivity in populations residing within Durango City, Durango, Mexico.

It is not unreasonable to think that people living near $\mathrm{CMC}$ may have been infected with T. gondii. Though no clinical cases of toxoplasmosis have been reported from bats, there have been reports describing the isolation of T. gondii from 2 species of bats (Vespertilio pipistrellus and Nyctalus noctula) in the USSR [60]. T. gondii is also maintained in a wide variety of wild animal populations, including rodents, carnivores, and lagomorphs [61-63]. This means that at least one type of reservoir host may have come into contact with people living near CMC. Congenital toxoplasmosis within humans may cause spontaneous abortions or fetal abnormalities. In modern cases of toxoplasmosis among healthy children, symptoms are few and most often fleeting. Children with weaken immune systems who contract toxoplasmosis are at risk for more serious neurological symptoms that may cause long-term damage. The cause of death among the children buried at CMC is not clear, but coprolite analyses show that children using CMC were likely malnourished and played hosts to a variety of parasites $[15,34,35]$. The high probability that the people occupying $\mathrm{CMC}$ were exposed to reservoir host species coupled with the majority of skeletons from this site being those of children make this site an excellent place to look for prehistoric toxoplasmosis.

Terra and colleagues [64] demonstrated through experimental desiccation of infected mice that PCR was effective in the recovery of T. gondii DNA; however, to date, there has been no archaeological evidence of this parasite recovered from any context. As is the case with T. cruzi, ELISA kits have been developed to test modern human serum for T. gondii antibodies rather than for parasite coproantigens in fecal samples. This makes such kits inefficient for recovering T. gondii evidence from coprolites. Fortunately, Hajeer and colleagues [22] found that $T$. gondii antibodies were detectible in modern saliva using ELISA kits developed for testing human serum. As with the theory presented for the recovery of T. cruzi evidence utilizing CMC quids, we predict that $T$. gondii evidence could be recovered using ELISA kits to test reconstituted quids. Despite the lack of evidence regarding prehistoric, New World toxoplasmosis, it is possible that those utilizing $\mathrm{CMC}$ may have contracted T. gondii. This parasite is known to infect a wide range of endothermic vertebrate hosts, including bats, rodents, and carnivores that may have come into contact with the Loma San Gabriel who were utilizing CMC. These individuals had close relationships with their companion canines, which could have been reservoirs for toxoplasmosis, and ate small rodents, rabbits, and other potential reservoir hosts that lived in the region surrounding the cave. Little is known about the paleoepidemiology of this parasite. If human antibodies created in response to T. gondii 1,300 years ago are capable of surviving and being detected using a commercially available ELISA kit, archaeoparasitologists would have a new means of tracing the origins of human toxoplasmosis in the New World. 


\section{MATERIALS AND METHODS}

The 45 CMC quids used for the present study were gathered from the collection of over 2,000 quids housed within the Pathoecology Laboratory in the School of Natural Resources at the University of Nebraska-Lincoln (Fig. 1; Table 1). To prevent modern contamination of material, nitrile gloves were worn throughout processing. A total of 45 quids were given a laboratory identification number (CMCQ-\#) for analysis and photographed using a Sony Cybershot 18.2 megapixel camera. Each of the quids was analyzed using an Olympus stereoscopic microscope and designated as being comprised of either Agave or non-Agave fibers. Other macrofossils, such as cordage and charcoal were also noted.

The quids were rehydrated in their entirety within a plastic, conical, $50 \mathrm{ml}$ centrifuge tube containing a $0.5 \%$ trisodium phosphate solution. Samples were rehydrated for $24 \mathrm{hr}$ before rehydration colors were recorded (Table 1). Following rehydration, the quids were disaggregated using a novel "shake and break" method. This method begins with vigorously shaking the capped centrifuge tube before vortexing the tube for a few seconds. Next, the material within the tube was poured through a $250 \mu \mathrm{m}$ mesh screen over a glass beaker. The tube was rinsed with a jet of distilled $\mathrm{H}_{2} \mathrm{O}$. Subsequently, sterile forceps were used in conjunction with a sterile spatulette to gently break apart the softened quids taking care to free as much of the non-fiber matrix of the quids as possible. Another jet of distilled $\mathrm{H}_{2} \mathrm{O}$ was applied before removing the fibers/macroscopic remains (items larger than $250 \mu \mathrm{m}$ ) on top of the screens to filter paper for drying. Microscopic remains (those smaller than $250 \mu \mathrm{m}$ ) were collected back into the centrifuge tubes and centrifuged to create the pellets used for ELISA testing.

Aliquots of processed quid material were assayed for human antibodies created in response to T. gondii and T. cruzi infections using commercially available antibody detection kits. ELISA kits were purchased from 2 suppliers: Sigma-Aldrich ${ }^{\circledR}$ (St. Louis, Missouri, USA) and Immuno-Biological Laboratories, Inc. [IBL-America] (Minneapolis, Minnesota, USA). The Toxoplasma IgA ELISA from Sigma-Aldrich ${ }^{\circledR}$ uses wells coated with a purified Toxoplasma antigen to bind with any Toxoplasma IgA specific antibodies present within a sample. Unbound materials are then washed away prior to the addition of an enzyme conjugate that binds to existing antibody-antigen complexes when present. An incubation step allows for oxidation of the substrate by the enzyme which invokes a color change in positive sample wells. The intensity of the color change is proportional to the amount of IgA specific antibody present within the sample (www.sigma-aldrich.com).

The Chagas (T. cruzi) IgG ELISA from Immuno-Biological Laboratories, Inc. [IBL-America] uses microtiter strip wells pre-
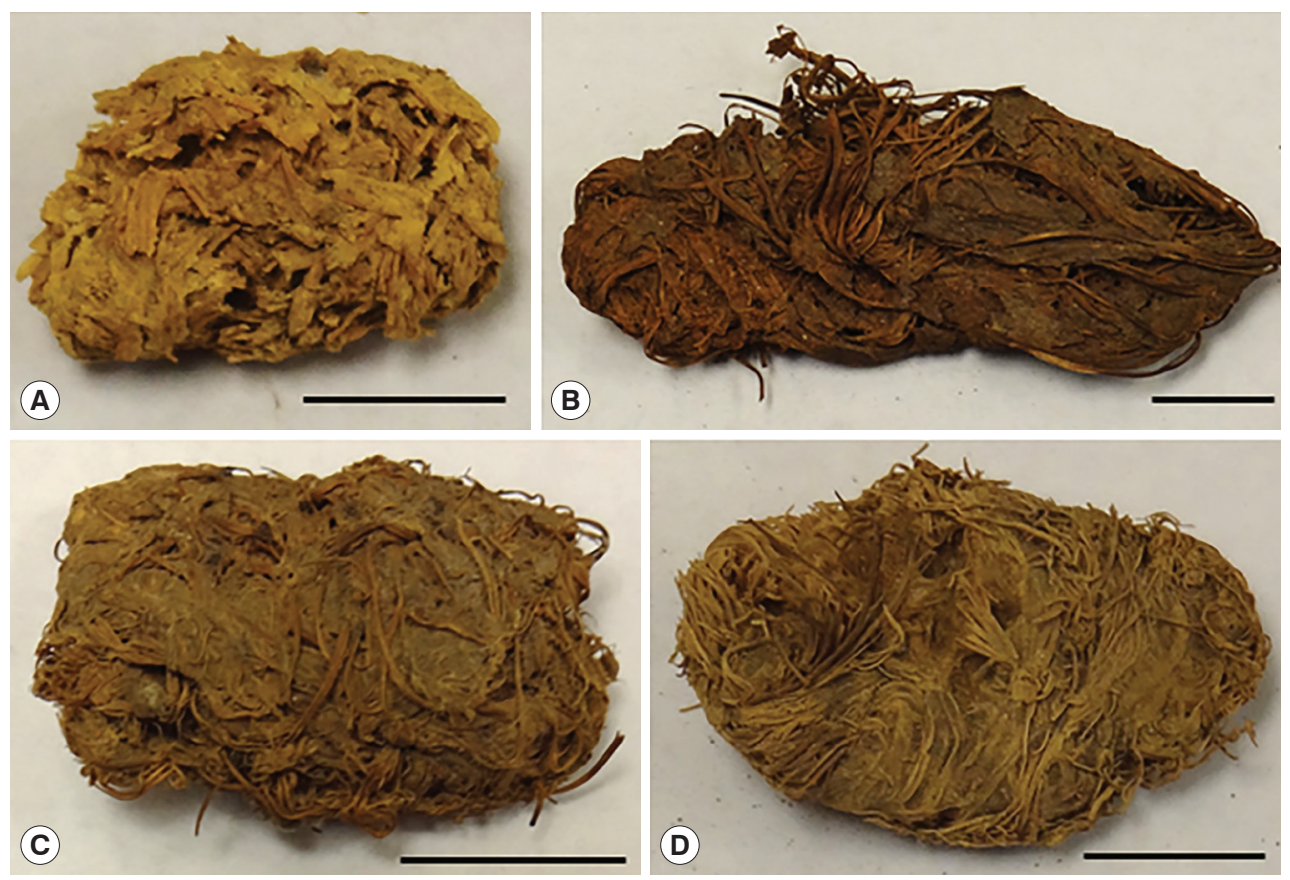

Fig. 1. Representative $C M C$ quids ( $A=C M C Q-37 ; B=C M C Q-20 ; C=C M C Q-4 ; D=C M C Q-21$ ) prior to rehydration. 
coated with Trypanosoma cruzi antigens to bind human IgGclass antibodies produced in response to T. cruzi infections. Samples are incubated to allow antigen-antibody complexes to form before washing procedures remove unbound materials from the sample wells. Next, a horseradish peroxidase

Table 1. Processing data for $\mathrm{CMC}$ quids

\begin{tabular}{|c|c|c|c|c|}
\hline \multicolumn{2}{|c|}{ Sample ID Provenience } & \multirow{2}{*}{$\begin{array}{l}\text { Level } \\
0-6\end{array}$} & \multirow{2}{*}{$\frac{\text { Weight }(\mathrm{g})}{1.4}$} & \multirow{2}{*}{$\begin{array}{c}\text { Rehydration color } \\
\text { dark brown }\end{array}$} \\
\hline CMCQ-1 & B2 & & & \\
\hline CMCQ-2 & $\mathrm{B} 2$ & $0-6$ & 1.3 & black \\
\hline CMCQ-3 & B2 & $6-12$ & 1.98 & black \\
\hline CMCQ-4 & $\mathrm{B} 2$ & $6-12$ & 1 & light brown \\
\hline CMCQ-5 & B3 & $0-4$ & 2.38 & black \\
\hline CMCQ-6 & B3 & $0-4$ & 0.92 & dark brown \\
\hline CMCQ-7 & B3 & $4-8$ & 1.84 & light brown \\
\hline CMCQ-8 & B3 & $4-8$ & 1.96 & medium brown \\
\hline CMCQ-9 & B3 & $8-12$ & 2.43 & dark brown \\
\hline CMCQ-10 & B3 & $16-20$ & 1.6 & black \\
\hline CMCQ-11 & B3 & $16-20$ & 2.05 & dark brown \\
\hline CMCQ-12 & B3 & $20-24$ & 1.8 & light brown \\
\hline CMCQ-13 & B3 & $20-24$ & 1.81 & brownish-red \\
\hline CMCQ-14 & B4 & 4-8 & 0.88 & amber \\
\hline CMCQ-15 & B4 & $4-8$ & 0.9 & black \\
\hline CMCQ-16 & B4 & $8-12$ & 1.55 & dark brown \\
\hline CMCQ-17 & B4 & $8-12$ & 1.75 & light brown \\
\hline CMCQ-18 & B4 & $12-16$ & 1.4 & light brown \\
\hline CMCQ-19 & B4 & $12-16$ & 1.3 & dark brown \\
\hline CMCQ-20 & B4 & $16-20$ & 2.19 & light brown \\
\hline CMCQ-21 & B4 & $20-24$ & 1.57 & amber \\
\hline CMCQ-22 & B4 & $20-24$ & 1.17 & light brown \\
\hline CMCQ-23 & B4 & $24-28$ & 1.47 & amber \\
\hline CMCQ-24 & B4 & $24-28$ & 1.49 & dark brown \\
\hline CMCQ-25 & B4 & $28-32$ & 0.7 & light brown \\
\hline CMCQ-26 & B4 & $28-32$ & 0.45 & amber \\
\hline CMCQ-27 & C1 & $4-8$ & 1.49 & dark brown \\
\hline CMCQ-28 & C1 & $4-8$ & 1.39 & dark brown \\
\hline CMCQ-29 & C1 & $8-12$ & 2.24 & black \\
\hline CMCQ-30 & C1 & $12-16$ & 0.56 & dark brown \\
\hline CMCQ-31 & $\mathrm{C} 2$ & $0-4$ & 2.04 & dark brown \\
\hline CMCQ-32 & $\mathrm{C} 2$ & $0-4$ & 1.3 & black \\
\hline CMCQ-33 & $\mathrm{C} 2$ & $8-12$ & 2.13 & black \\
\hline CMCQ-34 & $\mathrm{C} 2$ & $12-18$ & 0.65 & medium brown \\
\hline CMCQ-35 & $\mathrm{C} 2$ & $16-32$ & 1.26 & dark brown \\
\hline CMCQ-36 & D1 & $0-4$ & 0.45 & lightbrown \\
\hline CMCQ-37 & D1 & $4-8$ & 0.58 & medium brown \\
\hline CMCQ-38 & D1 & $8-12$ & 3.02 & amber \\
\hline CMCQ-39 & D1 & $12-16$ & 1.4 & light brown \\
\hline CMCQ-40 & D1 & $16-20$ & 0.58 & amber \\
\hline CMCQ-41 & D2 & $0-12$ & 1.35 & medium brown \\
\hline CMCQ-42 & $\mathrm{D} 2$ & $0-12$ & 0.81 & brownish-red \\
\hline CMCQ-43 & D2 & $12-16$ & 2.33 & light brown \\
\hline CMCQ-44 & D2 & $12-16$ & 0.5 & light brown \\
\hline CMCQ-45 & D2 & $16-20$ & 2.51 & black \\
\hline
\end{tabular}

(HRP) labelled Protein A conjugate is added to bind any antigen-antibody complexes present within the samples. The resultant immune complex that is formed is then visualized fol-

Table 2. Optical density (OD) values and sample designations for Toxoplasma gondii testing

\begin{tabular}{|c|c|c|c|c|}
\hline Sample ID & OD value \#1 & OD value \#2 & 2. Average OD & Designation \\
\hline Calibrator & 1.226 & 1.224 & 1.23 & $n / a^{a}$ \\
\hline Positive cut-off & 1.472 & 1.345 & 1.41 & $\mathrm{n} / \mathrm{a}$ \\
\hline Negative cut-off & 0.151 & 0.166 & 0.16 & $\mathrm{n} / \mathrm{a}$ \\
\hline CMCQ-1 & 0.084 & 0.118 & 0.10 & negative \\
\hline CMCQ-2 & 0.068 & 0.059 & 0.06 & negative \\
\hline CMCQ-3 & 0.074 & 0.077 & 0.08 & negative \\
\hline CMCQ-4 & 0.076 & 0.072 & 0.07 & negative \\
\hline CMCQ-5 & 0.113 & 0.072 & 0.09 & negative \\
\hline CMCQ-6 & 0.06 & 0.056 & 0.06 & negative \\
\hline CMCQ-7 & 0.062 & 0.062 & 0.06 & negative \\
\hline CMCQ-8 & 0.073 & 0.066 & 0.07 & negative \\
\hline CMCQ-9 & 0.069 & 0.069 & 0.07 & negative \\
\hline CMCQ-10 & 0.086 & 0.089 & 0.09 & negative \\
\hline CMCQ-11 & 0.085 & 0.094 & 0.09 & negative \\
\hline CMCQ-12 & 0.083 & 0.081 & 0.08 & negative \\
\hline CMCQ-13 & 0.083 & 0.07 & 0.08 & negative \\
\hline CMCQ-14 & 0.066 & 0.068 & 0.07 & negative \\
\hline CMCQ-15 & 0.073 & 0.091 & 0.08 & negative \\
\hline CMCQ-16 & 0.085 & 0.093 & 0.09 & negative \\
\hline CMCQ-17 & 0.109 & 0.092 & 0.10 & negative \\
\hline CMCQ-18 & 0.076 & 0.085 & 0.08 & negative \\
\hline CMCQ-19 & 0.088 & 0.078 & 0.08 & negative \\
\hline CMCQ-20 & 0.082 & 0.093 & 0.09 & negative \\
\hline CMCQ-21 & 0.083 & 0.087 & 0.09 & negative \\
\hline CMCQ-22 & 0.078 & 0.074 & 0.08 & negative \\
\hline CMCQ-23 & 0.074 & 0.072 & 0.07 & negative \\
\hline CMCQ-24 & 0.081 & 0.081 & 0.08 & negative \\
\hline CMCQ-25 & 0.081 & 0.073 & 0.08 & negative \\
\hline CMCQ-26 & 0.071 & 0.071 & 0.07 & negative \\
\hline CMCQ-27 & 0.076 & 0.078 & 0.08 & negative \\
\hline CMCQ-28 & 0.082 & 0.077 & 0.08 & negative \\
\hline CMCQ-29 & 0.084 & 0.107 & 0.10 & negative \\
\hline CMCQ-30 & 0.063 & 0.078 & 0.07 & negative \\
\hline CMCQ-31 & 0.068 & 0.068 & 0.07 & negative \\
\hline CMCQ-32 & 0.066 & 0.071 & 0.07 & negative \\
\hline CMCQ-33 & 0.063 & 0.068 & 0.07 & negative \\
\hline CMCQ-34 & 0.064 & 0.072 & 0.07 & negative \\
\hline CMCQ-35 & 0.076 & 0.084 & 0.08 & negative \\
\hline CMCQ-36 & 0.076 & 0.075 & 0.08 & negative \\
\hline CMCQ-37 & 0.076 & 0.072 & 0.07 & negative \\
\hline CMCQ-38 & 0.062 & 0.065 & 0.06 & negative \\
\hline CMCQ-39 & 0.069 & 0.071 & 0.07 & negative \\
\hline CMCQ-40 & 0.082 & 0.085 & 0.08 & negative \\
\hline CMCQ-41 & 0.072 & 0.076 & 0.07 & negative \\
\hline CMCQ-42 & 0.071 & 0.074 & 0.07 & negative \\
\hline CMCQ-43 & 0.079 & 0.078 & 0.08 & negative \\
\hline CMCQ-44 & 0.075 & 0.076 & 0.08 & negative \\
\hline CMCQ-45 & 0.075 & 0.072 & 0.07 & negative \\
\hline
\end{tabular}

${ }^{a}$ Not applicable. 
lowing the addition of tetramethylbenzidine (TMB) substrate. The intensity of the color change is proportional to the amount

Table 3. Optical density (OD) values and sample designations for Trypanosoma cruzi testing

\begin{tabular}{|c|c|c|c|c|}
\hline Sample ID & OD Value \#1 & OD Value \#2 & Average OD & Designation \\
\hline Substrate blank & 0.031 & 0.045 & 0.04 & $n / a^{a}$ \\
\hline Negative control & 0.106 & 0.14 & 0.12 & $\mathrm{n} / \mathrm{a}$ \\
\hline Positive control & 2.294 & 2.475 & 2.38 & $\mathrm{n} / \mathrm{a}$ \\
\hline Cut-off control & 0.719 & 0.719 & 0.72 & $\mathrm{n} / \mathrm{a}$ \\
\hline CMCQ-1 & 0.034 & 0.032 & 0.03 & negative \\
\hline CMCQ-2 & 0.103 & 0.029 & 0.07 & negative \\
\hline CMCQ-3 & 0.03 & 0.06 & 0.05 & negative \\
\hline CMCQ-4 & 0.035 & 0.035 & 0.04 & negative \\
\hline CMCQ-5 & 0.03 & 0.03 & 0.03 & negative \\
\hline CMCQ-6 & 0.037 & 0.045 & 0.04 & negative \\
\hline CMCQ-7 & 0.039 & 0.041 & 0.04 & negative \\
\hline CMCQ-8 & 0.041 & 0.043 & 0.04 & negative \\
\hline CMCQ-9 & 0.052 & 0.035 & 0.04 & negative \\
\hline CMCQ-10 & 0.035 & 0.035 & 0.04 & negative \\
\hline CMCQ-11 & 0.039 & 0.04 & 0.04 & negative \\
\hline CMCQ-12 & 0.043 & 0.041 & 0.04 & negative \\
\hline CMCQ-13 & 0.044 & 0.034 & 0.04 & negative \\
\hline CMCQ-14 & 0.037 & 0.029 & 0.03 & negative \\
\hline CMCQ-15 & 0.043 & 0.041 & 0.04 & negative \\
\hline CMCQ-16 & 0.046 & 0.059 & 0.05 & negative \\
\hline CMCQ-17 & 0.101 & 0.034 & 0.07 & negative \\
\hline CMCQ-18 & 0.126 & 0.035 & 0.08 & negative \\
\hline CMCQ-19 & 0.044 & 0.033 & 0.04 & negative \\
\hline CMCQ-20 & 0.051 & 0.091 & 0.07 & negative \\
\hline CMCQ-21 & 0.051 & 0.038 & 0.04 & negative \\
\hline CMCQ-22 & 0.036 & 0.034 & 0.04 & negative \\
\hline CMCQ-23 & 0.029 & 0.074 & 0.05 & negative \\
\hline CMCQ-24 & 0.036 & 0.041 & 0.04 & negative \\
\hline CMCQ-25 & 0.043 & 0.038 & 0.04 & negative \\
\hline CMCQ-26 & 0.028 & 0.032 & 0.03 & negative \\
\hline CMCQ-27 & 0.035 & 0.043 & 0.04 & negative \\
\hline CMCQ-28 & 0.041 & 0.044 & 0.04 & negative \\
\hline CMCQ-29 & 0.04 & 0.039 & 0.04 & negative \\
\hline CMCQ-30 & 0.04 & 0.056 & 0.05 & negative \\
\hline CMCQ-31 & 0.037 & 0.038 & 0.04 & negative \\
\hline CMCQ-32 & 0.037 & 0.049 & 0.04 & negative \\
\hline CMCQ-33 & 0.053 & 0.036 & 0.04 & negative \\
\hline CMCQ-34 & 0.036 & 0.034 & 0.04 & negative \\
\hline CMCQ-35 & 0.038 & 0.037 & 0.04 & negative \\
\hline CMCQ-36 & 0.127 & 0.047 & 0.09 & negative \\
\hline CMCQ-37 & 0.036 & 0.036 & 0.04 & negative \\
\hline CMCQ-38 & 0.039 & 0.034 & 0.04 & negative \\
\hline CMCQ-39 & 0.04 & 0.041 & 0.04 & negative \\
\hline CMCQ-40 & 0.047 & 0.086 & 0.07 & negative \\
\hline CMCQ-41 & 0.058 & 0.056 & 0.06 & negative \\
\hline CMCQ-42 & 0.04 & 0.035 & 0.04 & negative \\
\hline CMCQ-43 & 0.036 & 0.043 & 0.04 & negative \\
\hline
\end{tabular}

${ }^{a}$ Not applicable. of T. cruzi-specific IgG antibodies present within the samples. This kit has a specificity of 99\% and a sensitivity of $99 \%$ (www. ibl-america.com).

Quid samples were subjected to ELISA testing for T. gondii and T. cruzi evidence at the Zera Lab within the School of Biological Sciences at the University of Nebraska-Lincoln. Samples were tested in duplicate along with controls provided by the manufacturers following manufacturer instructions. A visual inspection was conducted following the addition of each kit's stop solution and optical density (OD) values were collected with the aid of an Omega ELISA plate reader (Tables 2, 3).

The OD readings were used to determine the presence or absence of each parasite using the interpretational procedures outlined for each kit by the manufacturers (Tables 2, 3). For the Toxoplasma IgA ELISA, the kit-specific Calibration Factor was multiplied by the OD value for the calibrator provided (must be greater than 0.250 to be considered valid) to determine the cut-off value. The $\mathrm{Ab}$ (antibody) Index was then calculated by dividing the OD value of the provided positive control by the cut-off value. The Ab Index was similarly calculated for the provided negative control and for each quid sample. Samples with an Ab Index less than 0.9 were designated as being negative while samples with an $\mathrm{Ab}$ Index greater than 1.2

Table 4. Processing data for quids utilized in secretory $\lg A(\operatorname{sig} A)$ testing

\begin{tabular}{lcccl}
\hline Sample ID & Provenience & Level & Weight (g) & Rehydration color \\
\hline CMCQ-3 & B2 & $6-12$ & 1.98 & black \\
CMCQ-5 & B3 & $0-4$ & 2.38 & black \\
CMCQ-13 & B3 & $20-24$ & 1.81 & brownish-red \\
CMCQ-16 & B4 & $8-12$ & 1.55 & dark brown \\
CMCQ-21 & B4 & $20-24$ & 1.57 & amber \\
CMCQ-24 & B4 & $24-28$ & 1.49 & dark brown \\
CMCQ-29 & C1 & $8-12$ & 2.24 & black \\
CMCQ-35 & C2 & $16-32$ & 1.26 & dark brown \\
CMCQ-37 & D1 & $4-8$ & 0.58 & medium brown \\
CMCQ-39 & D1 & $12-16$ & 1.4 & light brown \\
CMCQ-43 & D2 & $12-16$ & 2.33 & light brown \\
CMCQ-46 & B2 & $0-6$ & 0.53 & black \\
CMCQ-47 & B2 & $6-12$ & 0.46 & light brown \\
CMCQ-48 & B3 & $4-8$ & 0.59 & light brown \\
CMCQ-49 & B3 & $12-16$ & 0.76 & medium brown \\
CMCQ-50 & B3 & $16-20$ & 1.05 & amber; opaque \\
CMCQ-51 & B3 & $20-24$ & 1.13 & light brown \\
CMCQ-52 & B4 & $16-20$ & 1.71 & amber \\
CMCQ-53 & B4 & $24-28$ & 0.87 & yellow-gray \\
CMCQ-54 & C2 & $0-4$ & 0.71 & black \\
CMCQ-55 & D2 & $0-12$ & 0.37 & light brown \\
CMCQ-56 & D2 & $12-16$ & 0.15 & light brown \\
\hline & & & & \\
\hline
\end{tabular}


were deemed to be considered positive. Samples with Ab Indices between 0.9 and 1.2 were considered borderline positive and would require follow-up testing for validation (Table 2).

The cut-off value for the Chagas (T. cruzi) IgG ELISA was calculated by taking the average of the OD values for the cut-off control wells. Substrate blank-corrected sample OD values less than the cut-off were considered negative, while values greater than the cut-off were considered positive. For the assay to be considered valid, cut-off values had to range between 0.150 and 1.30 prior to blank correction (Table 3).

Following these analyses, it became apparent that further method development would be necessary to validate interpretations. To facilitate this, sIgA ELISA test kit from Eagle Biosciences, Inc. was utilized. A total of $11 \mathrm{CMC}$ quids processed for the tests described above were chosen to represent many different proveniences and depths (CMCQ\#s: 3, 5, 13, 16, 24, 29,

Table 5. Optical density (OD) values for slgA testing of undiluted quid samples

\begin{tabular}{lcccc}
\hline Sample ID & OD value \#1 & OD value \#2 & Average OD & Designation \\
\hline Control 1 & 0.272 & $\mathrm{n} / \mathrm{a}^{\mathrm{a}}$ & $\mathrm{n} / \mathrm{a}$ & $\mathrm{n} / \mathrm{a}$ \\
Control 2 & 0.852 & $\mathrm{n} / \mathrm{a}$ & $\mathrm{n} / \mathrm{a}$ & $\mathrm{n} / \mathrm{a}$ \\
Standard 0 & 0.064 & $\mathrm{n} / \mathrm{a}$ & $\mathrm{n} / \mathrm{a}$ & $\mathrm{n} / \mathrm{a}$ \\
Standard 22.2 & 0.209 & $\mathrm{n} / \mathrm{a}$ & $\mathrm{n} / \mathrm{a}$ & $\mathrm{n} / \mathrm{a}$ \\
Standard 66.6 & 0.256 & $\mathrm{n} / \mathrm{a}$ & $\mathrm{n} / \mathrm{a}$ & $\mathrm{n} / \mathrm{a}$ \\
Standard 200 & 0.54 & $\mathrm{n} / \mathrm{a}$ & $\mathrm{n} / \mathrm{a}$ & $\mathrm{n} / \mathrm{a}$ \\
Standard 600 & 1.157 & $\mathrm{n} / \mathrm{a}$ & $\mathrm{n} / \mathrm{a}$ & $\mathrm{n} / \mathrm{a}$ \\
0.5 \% TSP & 0.161 & $\mathrm{n} / \mathrm{a}$ & $\mathrm{n} / \mathrm{a}$ & n/a \\
CMCQ-3 & 0.081 & 0.05 & 0.0655 & negative \\
CMCQ-5 & 0.06 & 0.055 & 0.0575 & negative \\
CMCQ-13 & 0.068 & 0.06 & 0.064 & negative \\
CMCQ-16 & 0.054 & 0.191 & 0.1225 & negative \\
CMCQ-21 & 0.135 & 0.059 & 0.097 & negative \\
CMCQ-24 & 0.101 & 0.073 & 0.087 & negative \\
CMCQ-29 & 0.104 & 0.076 & 0.09 & negative \\
CMCQ-35 & 0.067 & 0.063 & 0.065 & negative \\
CMCQ-37 & 0.051 & 0.076 & 0.0635 & negative \\
CMCQ-39 & 0.055 & 0.061 & 0.058 & negative \\
CMCQ-43 & 0.053 & 0.102 & 0.0775 & negative \\
CMCQ-46 & 0.064 & 0.066 & 0.065 & negative \\
CMCQ-47 & 0.057 & 0.057 & 0.057 & negative \\
CMCQ-48 & 0.135 & 0.07 & 0.1025 & negative \\
CMCQ-49 & 0.059 & 0.121 & 0.09 & negative \\
CMCQ-50 & 0.043 & 0.066 & 0.0545 & negative \\
CMCQ-51 & 0.087 & 0.113 & 0.1 & negative \\
CMCQ-52 & 0.1 & 0.059 & 0.0795 & negative \\
\hline CMCQ-53 & 0.059 & 0.065 & 0.062 & negative \\
CMCQ-54 & 0.056 & 0.112 & 0.084 & negative \\
CMCQ-55 & 0.083 & 0.065 & 0.074 & negative \\
CMCQ-56 & 0.109 & 0.099 & 0.104 & negative \\
\hline & & & &
\end{tabular}

${ }^{a}$ Not applicable.
$35,37,39$, and 43 ). Additionally, 11 previously unprocessed quids were selected from the $\mathrm{CMC}$ collection held within the Pathoecology Laboratory at UNL's School of Natural Resources. These quids were weighed, photographed, rehydrated in $0.5 \%$ trisodium phosphate, and further processed as previously described (Table 4).

Aliquots from each of these 22 samples were transferred into pre-labeled microcentrifuge tubes. A second aliquot of precisely $10 \mu$ from each sample was placed into microcentrifuge tubes along with $990 \mu \mathrm{l}$ of wash buffer to test whether or not dilution of samples made a difference in the recovery of antibodies using these techniques. All samples were run in duplicate for both their diluted and undiluted varieties (Tables 5-7).

The sIgA ELISA from Eagle Biosciences, Inc. uses a pre-coated microtiter plate to bind sIgA within samples, standards, and controls. After an initial wash, a peroxidase-labeled detection

Table 6. Optical density (OD) values for slgA testing of diluted quid samples

\begin{tabular}{lcccc}
\hline Sample ID & OD value \#1 & OD value \#2 & Average OD & Designation \\
\hline Control 1 & 0.272 & $\mathrm{n} / \mathrm{a}^{\mathrm{a}}$ & $\mathrm{n} / \mathrm{a}$ & $\mathrm{n} / \mathrm{a}$ \\
Control 2 & 0.852 & $\mathrm{n} / \mathrm{a}$ & $\mathrm{n} / \mathrm{a}$ & $\mathrm{n} / \mathrm{a}$ \\
Standard 0 & 0.064 & $\mathrm{n} / \mathrm{a}$ & $\mathrm{n} / \mathrm{a}$ & $\mathrm{n} / \mathrm{a}$ \\
Standard 22.2 & 0.209 & $\mathrm{n} / \mathrm{a}$ & $\mathrm{n} / \mathrm{a}$ & $\mathrm{n} / \mathrm{a}$ \\
Standard 66.6 & 0.256 & $\mathrm{n} / \mathrm{a}$ & $\mathrm{n} / \mathrm{a}$ & $\mathrm{n} / \mathrm{a}$ \\
Standard 200 & 0.54 & $\mathrm{n} / \mathrm{a}$ & $\mathrm{n} / \mathrm{a}$ & $\mathrm{n} / \mathrm{a}$ \\
Standard 600 & 1.157 & $\mathrm{n} / \mathrm{a}$ & $\mathrm{n} / \mathrm{a}$ & $\mathrm{n} / \mathrm{a}$ \\
0.5 \% TSP & 0.161 & $\mathrm{n} / \mathrm{a}$ & $\mathrm{n} / \mathrm{a}$ & n/a \\
CMCQ-3 & 0.123 & 0.113 & 0.118 & negative \\
CMCQ-5 & 0.06 & 0.055 & 0.0575 & negative \\
CMCQ-13 & 0.051 & 0.157 & 0.104 & negative \\
CMCQ-16 & 0.083 & 0.132 & 0.1075 & negative \\
CMCQ-21 & 0.066 & 0.097 & 0.0815 & negative \\
CMCQ-24 & 0.079 & 0.127 & 0.103 & negative \\
CMCQ-29 & 0.123 & 0.057 & 0.09 & negative \\
CMCQ-35 & 0.083 & 0.238 & 0.1605 & negative \\
CMCQ-37 & 0.111 & 0.128 & 0.1195 & negative \\
CMCQ-39 & 0.082 & 0.096 & 0.089 & negative \\
\hline CMCQ-43 & 0.067 & 0.085 & 0.076 & negative \\
CMCQ-46 & 0.108 & 0.111 & 0.1095 & negative \\
CMCQ-47 & 0.095 & 0.072 & 0.0835 & negative \\
CMCQ-48 & 0.062 & 0.057 & 0.0595 & negative \\
CMCQ-49 & 0.134 & 0.064 & 0.099 & negative \\
CMCQ-50 & 0.055 & 0.034 & 0.0445 & negative \\
\hline CMCQ-51 & 0.103 & 0.039 & 0.071 & negative \\
CMCQ-52 & 0.09 & 0.046 & 0.068 & negative \\
CMCQ-53 & 0.06 & 0.038 & 0.049 & negative \\
CMCQ-54 & 0.185 & 0.084 & 0.1345 & negative \\
CMCQ-55 & 0.061 & 0.043 & 0.052 & negative \\
CMCQ-56 & 0.07 & 0.073 & 0.0715 & negative \\
\hline
\end{tabular}

${ }^{a}$ Not applicable. 
Table 7. Optical density (OD) values for slgA testing of undiluted quid samples

\begin{tabular}{lccc}
\hline Sample ID & $\begin{array}{c}\text { Undiluted samples } \\
\text { (average OD) }\end{array}$ & $\begin{array}{c}\text { Diluted samples } \\
\text { (average OD) }\end{array}$ & Higher OD \\
\hline CMCQ-3 & 0.0655 & 0.118 & diluted \\
CMCQ-5 & 0.0575 & 0.0575 & same \\
CMCQ-13 & 0.064 & 0.104 & diluted \\
CMCQ-16 & 0.1225 & 0.1075 & undiluted \\
CMCQ-21 & 0.097 & 0.0815 & undiluted \\
CMCQ-24 & 0.087 & 0.103 & diluted \\
CMCQ-29 & 0.09 & 0.09 & same \\
CMCQ-35 & 0.065 & 0.1605 & diluted \\
CMCQ-37 & 0.0635 & 0.1195 & diluted \\
CMCQ-39 & 0.058 & 0.089 & diluted \\
CMCQ-43 & 0.0775 & 0.076 & undiluted \\
CMCQ-46 & 0.065 & 0.1095 & diluted \\
CMCQ-47 & 0.057 & 0.0835 & diluted \\
CMCQ-48 & 0.1025 & 0.0595 & undiluted \\
CMCQ-49 & 0.09 & 0.099 & same \\
CMCQ-50 & 0.0545 & 0.0445 & undiluted \\
CMCQ-51 & 0.1 & 0.071 & undiluted \\
CMCQ-52 & 0.0795 & 0.068 & undiluted \\
CMCQ-53 & 0.062 & 0.049 & undiluted \\
CMCQ-54 & 0.084 & 0.1345 & diluted \\
CMCQ-55 & 0.074 & 0.052 & undiluted \\
CMCQ-56 & 0.104 & 0.0715 & undiluted \\
\hline
\end{tabular}

antibody is added, which binds to the antibody complex. A second wash is performed before adding a substrate, which is converted by the peroxidase to result in a colored product. The addition of an acidic stop solution terminates this reaction and optical densities are read at $450 \mathrm{~nm}$. The optical densities are then used to calculate sIgA concentrations with the help of a standard curve (www.eaglebio.com). For the purposes of this study, sIgA concentrations were not calculated as a simple presence/absence test was all that was needed to test the methods.

\section{RESULTS}

Visual inspection of the ELISA plates was conducted prior to the collection of OD values via the ELISA plate reader. Positive/negative assessments were recorded on the basis of a visual color change and later compared to sample designations using OD values. All sample wells across both test kits were considered negative via visual inspection.

Data collected via the ELISA plate reader also yielded negative results for both test kits. The OD values from these analyses were recorded and samples were designated positive/negative according to cut-off value calculations using manufactur- ers' interpretational protocols (Tables 2, 3).

Visual inspection designations were in 100\% agreement with sample designations determined using OD values. All controls used in both kits yielded OD values within acceptable ranges for considering the kits valid according to manufacturers' specifications.

A visual inspection of the sIgA ELISA wells produced 2 positive test wells from CMCQ-35 and CMCQ-49 (both diluted). However, the duplicates of both wells were visually negative. Upon examination of OD values, only CMCQ-35 remained positive, again with its duplicate well being negative. Because duplicate wells for both samples were not consistently positive and because average OD values led to negative designations for both samples, these were considered anomalies rather than true positive results.

Because the sIgA ELISA kit used was developed for determining concentration levels of sIgA in modern samples and not for determining presence/absence of sIgA in archaeological samples, positive/negative determinations were reached as follows. The OD value for $0.5 \%$ trisodium phosphate $(0.161)$ was subtracted from each sample $\mathrm{OD}$ value (i.e. the $\mathrm{OD}$ value for the rehydration solution was used as a blank). Blank-corrected OD values were then compared to the OD values acquired for the kit-provided standards. None of the OD values were close to the higher $\mathrm{ng} / \mu \mathrm{l}$ standards (i.e. the $200 \mathrm{ng} / \mu \mathrm{l}$ standard and the $600 \mathrm{ng} / \mu \mathrm{l}$ standard), so OD values were instead compared to the OD values for the $22.2 \mathrm{ng} / \mu \mathrm{l}$ standard and the $0 \mathrm{ng} / \mu \mathrm{l} \mathrm{stan}$ dard. Blank-corrected OD values greater than the OD value for the $0 \mathrm{ng} / \mu \mathrm{l}$ standard (0.064) were considered potentially positive. Average OD values calculated rom sample duplicates were also compared to the OD value for the $0 \mathrm{ng} / \mu \mathrm{l}$ standard (0.064) and the entire quid was considered potentially positive if greater than 0.064 . Similar comparisons were made using the OD values for the $22.2 \mathrm{ng} / \mu \mathrm{l}$ standard (0.209). Samples with $O D$ values and OD value averages greater than 0.209 were considered positive for trace amounts of sIgA. Only a single test well (CMCQ-35--diluted) was deemed potentially positive in this way. However, since the duplicate for this well was negative and the average OD value for this quid yielded a negative designation, this was not found to be significant and was likely a false positive as a result of an error in processing. All other sample wells were found to be negative when compared to OD readings for the standards.

The average OD values for each sample in their diluted and undiluted forms were compared. Out of the 22 samples tested, 
3 produced the same OD value whether or not they were diluted. A total of 9 samples yielded higher OD values for diluted samples while a total of 10 samples yielded higher OD values for undiluted samples (Table 7).

\section{DISCUSSION}

While other studies have successfully employed ELISA techniques for recovering parasite evidence from latrine and burial sediments $[3,4,10]$ as well as coprolites $[2,14,15]$, none have successfully recovered parasite antigens or parasite-induced antibodies from quids. Although the present study was not successful in the recovery of parasite-induced antibodies from CMC quids, the study represents the first attempt to use quids as a source material for archaeoparasitological investigations.

There are several reasons why this study's attempt at the recovery of parasite evidence from quids may have failed. Despite the recovery of $T$. gondii and T. cruzi antibodies from modern saliva, it is important to note that ELISA kits available for $T$. gondii and T. cruzi are marketed for use with human serum. Furthermore, these kits are marketed for modern material and not for reconstituted archaeological material, which could compromise the abilities of the kits to actually detect ancient antibodies. This has not been a problem for the recovery of parasite coproantigens out of archaeological materials, but antibodies may degrade differently than do coproantigens. The negative results of the subsequent sIgA ELISA tests support this. Antibodies are much more structurally complex and heavier than antigens. These molecules weigh about $150 \mathrm{kDa}$ as compared to the average antigen weighing in at around 14 $\mathrm{kDa}$. The smaller size and relative structural simplicity of antigens make them less likely to degrade in ways that would render ELISA testing inefficient. It is unknown whether or not plant products, such as those made by Agave and other types of botanicals found in quids, have an adverse effect on the preservation of antibodies and antigens. If so, such products could also contribute to antibody degradation, which further complicates the ELISA testing of quids. The archaeological context, structural components of antibodies/antigens, anthropogenic behaviors regarding quid chewing, and ecological interactions of plants with target antibodies/antigens may all contribute to the taphonomy of immunological parasite evidence in much the same way that similar factors contribute to the degradation of physical parasite eggs within archaeological contexts as described by Morrow and colleagues [65]. Even so, the taphonomic impacts of archaeological antibody/antigen recovery have not been thoroughly assessed at the present time. Future examinations of antibody degradation resulting from desiccation could elucidate the nature of human antibody taphonomy and its implications on archaeoparasitological recovery techniques.

Alternatively, the capabilities of the ELISA test kits utilized in the present study could be sound for the recovery of archaeological antibodies given the proper quid processing methods. Without precedent in the existing literature, quid processing methods were developed by modifying coprolite processing techniques deemed successful for the recovery of parasite coproantigens [15]. Further refinement of quid processing methods could provide avenues for the successful recovery of parasite evidence utilizing ELISA kits.

Finally, the results of these analyses could have been negative simply because the individuals who created the CMC quids were not infected with T. gondii or T. cruzi. The pathoecological potential of parasitism does not always reflect true parasitism within a given population. The individuals utilizing $\mathrm{CMC}$ could have circumvented entry into zoonotic cycles of these parasites by avoiding transmission pathways. Transmission pathway avoidance could have been a result of cultural behaviors that limited infection risks. The coprolites of these individuals contained large quantities of dietary fiber $[18,20]$, which may reflect a greater reliance on plant foodstuffs than on wild game. Although these people were known to ingest rodents, perhaps the animals they were consuming were not serving as reservoir hosts for either of these parasites around $\mathrm{CMC}$ at that time.

Despite the lack of positive results, the employment of ELISA for the recovery of parasite evidence using quids as source materials remains a plausible concept. Future ELISA tests of quids should target parasite antigens as opposed to parasiteinduced human antibodies, though currently, few such tests have been developed for samples of non-fecal origin. Future researchers should approach this concept by modifying processing methods, experimenting with different epitope targets and brands of ELISA kits, and performing methodological experiments using artificially-created quids known to contain target antibodies. Such experimentation could provide the foundational data needed to develop reliable techniques for recovering parasite evidence from quids.

The development of successful methods for the archaeoparasitological analysis of quids would open new venues of re- 
search regarding ancient parasitism. Potentially, parasites that do not leave behind traceable coproantigens, such as T. gondii and $T$. cruzi, could be detected via residual salivary products present in artifacts like quids. As researchers move forward with the analysis of quids, it is important to consider the limitations of ELISA testing. Such limitations include taphonomic degradation of target antibodies/antigens within quids, crossreactivity potential with other parasites, and the presence of bacteria or fungi that may compromise test validity. To address these limitations, processing methods should be designed to inflict little damage to potential antibodies/antigens, only kits that are highly specific/sensitive should be employed, and samples should be tested quickly after being reconstituted before bacterial and fungi are able to colonize samples. It is also vital that future researchers acknowledge that archaeoparasitological data do not reflect actual infection rates in past populations with $100 \%$ accuracy. Nonetheless, immunological testing of archaeological samples provides valuable tools for estimating the parasite burdens among peoples of the past. Our ability to more accurately assess protozoan parasitism of the past has improved with the progression of technology and will continue to be refined as new archaeoparasitological immunodiagnostic techniques become available.

\section{ACKNOWLEDGMENTS}

The authors thank Dr. Anthony Zera for sharing access to his laboratory, refrigerator space, and Omega ELISA plate reader. We also thank Abigail Neyer, Ruth Grady, and Becca Bevans for all of their assistance and Dr. Gwen Bachman for access to her laboratory during ELISA processing. We thank Christian Elowsky for his valuable contributions to the Toxoplasma gondii research. We thank Eagle Biosciences for their generous donation of the sIgA salivary ELISA kit.

\section{CONFLICT OF INTEREST}

We have no conflict of interest related to this work.

\section{REFERENCES}

1. Allison MJ, Bergman T, Gerszten E. Further studies on fecal parasites in antiquity. Am J Clin Pathol 1999; 112: 605-609.

2. Gonçalves MLC, Araújo A, Duarte R, da Silva JP, Reinhard KJ, Bouchet F, Ferreira LF. Detection of Giardia duodenalis antigen in coprolites using a commercially available enzyme-linked immu- nosorbent assay. Trans Roy Soc Trop Med Hyg 2002; 96: 640643.

3. Gonçalves MLC, Araújo A, Ferreira LF. Human intestinal parasites in the past: new findings and a review. Mem Inst Oswaldo Cruz 2003; 98(suppl 1): 103-118.

4. Gonçalves MLC, da Silva VL, de Andrade CM, Reinhard K, da Rocha GC, Le Bailly M, Bouchet F, Ferreira LF, Araújo A. Amoebiasis distribution in the past: first steps using an immunoassay technique. Trans Roy Soc Trop Med Hyg 2004; 98: 88-91.

5. Gonçalves MLC, Schnell C, Sianto L, Bouchet F, Le Bailly M, Reinhard K, Ferreira LF, Araújo A. Protozoan infection in archaeological material. J Bio Res 2005; 80: 146-148.

6. Ortega YR, Bonavia D. Cryptosporidium, Giardia and Cyclospora in ancient Peruvians. J Parasitol 2003; 89: 635-636.

7. Le Bailly M, Bouchet F. Paléoparasitologie et immunologie: l'exemple d'Entamoeba histolytica. Revue Archéométrie 2006; 30: 129-135.

8. Le Bailly M, Gonçalves MLC, Lefevre C, Roper D, Pye JW, Araújo A, Bouchet F. Parasitism in Kansas in the 1800s - A glimpse to the past through the analysis of grave sediments from Meadowlark cemetery. Mem Inst Oswaldo Cruz 2006; 101: 53-56.

9. Le Bailly M, Gonçalves MLC, Harter-Lailheugue S, Prodéo F, Araújo A, Bouchet F. New finding of Giardia intestinalis (Eukaryote, Metamonad) in Old World archaeological site using immunofluorescence and enzyme-linked immunosorbent assays. Mem Inst Oswaldo Cruz 2008; 103: 298-300.

10. Le Bailly M, Romon Th, Kacki S. New evidence of Entamoeba histolytica infections in pre-Columbian and colonial cemeteries in the Caribbean. J Parasitol 2014; 100: 684-686.

11. Mitchell PD, Stern E, Tepper Y. Dysentery in the crusader kingdom of Jerusalem: an ELISA analysis of two medieval latrines in the city of Acre (Israel). J Archaeol Sci 2008; 35: 1849-1853.

12. Frías L, Leles D, Araújo A. Studies on protozoa in ancient remains-a review. Mem Inst Oswaldo Cruz 2013; 108: 1-12.

13. Yeh HY, Pluskowski A, Kalëjs U, Mitchell PD. Intestinal parasites in a mid-14th century latrine from Riga, Latvia: fish tapeworm and the consumption of uncooked fish in the medieval eastern Baltic region. J Archaeol Sci 2014; 49: 83-89.

14. Yeh HY, Prag K, Clamer C, Humbert JB, Mitchell PD. Human intestinal parasites from a Mamluk Period cesspool in the Christian quarter of Jerusalem: Potential indicators of long distance travel in the 15th century AD. Int J Paleopathology 2015; 9: 69-75.

15. Morrow JJ, Reinhard KJ. Cryptosporidium parvum among coprolites from La Cueva de los Muertos Chiquitos (600-800 AD), Rio Zape Valley, Durango, Mexico. J Parasitol 2016; 102: 429435.

16. Turner C. Bite-marks in tule quids of prehistoric Nevada Indians. Rep Uni Cali Archaeol Surv 1967; 70: 1-1.

17. Reed AD. An analysis of the quids from Hoy House and Lion House. SW Lore. 1978; 44: 1-22.

18. Danielson DR, Reinhard KJ. Human dental microwear caused by calcium oxalate phytoliths in prehistoric diet of the lower Pecos region, Texas. Am J Phys Anthropol 1998; 107: 297-304. 
19. Hammerl EE, Baier MA, Reinhard KJ. Agave chewing and dental wear: evidence from quids. PLoS One 2015; 10: e0133710.

20. Meade T. A dietary analysis of coprolites from a prehistoric Mexican cave site. Thesis. University of Nebraska-Lincoln. USA. 1994.

21. Reinhard KJ, Danielson DR. Pervasiveness of phytoliths in prehistoric southwestern diet and implications for regional and temporal trends for dental microwear. J Archaeol Sci 2005; 32: 981-988.

22. Hajeer AH, Balfour AH, Mostratos A, Crosse B. Toxoplasma gondii: detection of antibodies in human saliva and serum. Parasite Immunol 1994; 16: 43-50.

23. Pinho RT, Pedrosa RC, Costa-Martins P, Castello-Branco LRR. Saliva ELISA: a method for the diagnosis of chronic Chagas disease in endemic areas. Acta Trop 1999; 72: 31-38.

24. Morris M., Cohen B, Andrews N, Brown D. Stability of total and rubellaspecific IgG in oral fluid samples: the effect of time and temperature. J Immunol Methods 2002; 266: 211-216.

25. Garde AH, Hansen HM. Long-term stability of salivary cortisol. Scand J Clin Lab Invest 2005; 65: 433-436.

26. Kelley JC. Settlement patterns in North-Central Mexico. In GR Willey ed, Prehistoric settlement patterns in the New World. New York, New York, USA. Viking Fund Publications in Anthropology. 1956, pp 128-129.

27. Kelley JC. Archaeology of the northern frontier: Zacatecas and Durango. In Ekholm GF, Bernal I eds, Handbook of Middle American Indians, Vol. 11. Archaeology of Northern Mesoamerica, Part 2. Austin, Texas, USA. University of Texas Press. 1971, pp 768-801.

28. Brooks ST, Brooks RH. Cranial deformation: possible evidence of Pochteca trading movements. Tran Illinois State Aca Sci 1980; 72: 1-12.

29. Brooks RH, Kaplan L, Cutler HC, Whitaker TW. Plant material from a cave on the Rio Zape, Durango, Mexico. Am Antiquity 1962; 27: 356-369.

30. Foster MS. The Weicker site: a Loma San Gabriel hamlet in Durango, Mexico. J Field Archaeol 1986; 13: 7-19.

31. Brooks ST, Brooks RH. Paleoepidemiology as a possible interpretation of multiple child burials near Zape Chico, Durango, Mexico. In Kelley JC, Riley CL, Hedrick BC eds, Across the Chichimec Sea: Papers in honor of J. Charles Kelley. Carbondale, Illinois, USA. Southern Illinois University Press. 1978, pp 96-101.

32. Phillips DA Jr. Prehistory of Chihuahua and Sonora, Mexico. J World Prehist 1989; 3: 373-401.

33. Foster MS. Loma San Gabriel subsistence patterns: a preliminary discussion. Anthropology 1984; 8: 13-30.

34. Jiménez FA, Gardner SL, Araújo A, Fugassa MH, Brooks RH, Rácz E, Reinhard KJ. Zoonotic and human parasites of inhabitants of La Cueva de Los Muertos Chiquitos, Rio Zape Valley, Durango, Mexico. J Parasitol 2012; 98: 304-309.

35. Tito RY, Knights D, Metcalf J, Obregon-Tito AJ, Cleeland L, Najar F, Roe B, Reinhard K, Sobolik K, Belknap S, Foster M, Spicer P, Knight R, Lewis CM Jr. Insights from characterizing extinct human gut microbiomes. PLoS One 2012; 7: e51146.
36. Cleeland L, Reichard MV, Tito RY, Reinhard KJ, Lewis CM. Clarifying prehistoric parasitism from a complementary morphological and molecular approach. J Archaeol Sci 2013; 40: 3060-3066.

37. Ferreira LF, Britto C, Cardoso A, Fernandes O, Reinhard K, Araújo A. Paleoparasitology of Chagas disease revealed by infected tissues of Chilean mummies. Acta Trop 2000; 7: 79-84.

38. Reinhard KJ, Fink TM, Skiles J. A case of megacolon in the Rio Grande Valley as a possible case of Chagas disease. Mem Inst Oswaldo Cruz 2003; 98: 165-172.

39. Aufderheide AC, Wilmar S, Madden M, Streitz J, Buikstra J, Guhl F, Arriaza B, Renier C, Wittmers LE Jr., Fornaciari G, Allison M. A 9,000-year record of Chagas' disease. Proc Natl Acad Sci USA 2004; 101: 2034-2039.

40. Aufderheide AC, Salo W, Madden M, Streitz IM, de la Cruz KD, Buikstra JE, Arriaza B, Wittmers LE. Aspects of ingestion transmission of Chagas disease identified in mummies and their coprolites. Chungara 2005; 37: 85-90.

41. Fernandes A, Iñiguez AM, Lima VS, Souza SM, Ferreira LF, Vicente AC, Jansen AM. Pre-columbian Chagas disease in Brazil: T. cruzi I in the archaeological remains of a human in Peruaçu Valley, Minas Gerais, Brazil. Mem Inst Oswaldo Cruz 2008; 103: 514-516.

42. Araújo A, Jansen AM, Reinhard K, Ferreira LF. Paleoparasitology of Chagas disease: a review. Mem Inst Oswaldo Cruz 2009; 104: 9-16.

43. Ferreira LF, Jansen AM, Araújo A. Chagas disease in prehistory. An Acad Bras Cienc 2011; 83: 1041-1044.

44. Reinhard KJ, Araújo A. Prehistoric earth oven facilities and the pathoecology of Chagas disease in the Lower Pecos Canyonlands. J Archaeol Sci 2015; 53: 227-234.

45. Herrera L. Una revisión sobre reservorios de Trypanosoma (Schizotrypanum) cruzi (Chagas, 1909), agente etiológico de la Enfermedad de Chagas. Bol Mal Sal Amb 2010; L: 1-13.

46. Reinhard KJ, Szuter C, Ambler JR. Hunter-gatherer use of small animal food resources. Int J Osteoarchaeol 2007; 17: 416-428.

47. Bonney KM. Chagas disease in the 21st century: a public health success or an emerging threat? Parasite 2014; 21: 11.

48. Rothhammer F, Allison MJ, Nuñez L, Staden V, Arriaza B. Chagas disease in pre-Columbian South America. Am J Phys Anthropol 1985; 68: 495-498.

49. Fornaciari G, Castagna M, Viacava P, Tognetti A, Bevilacqua G, Segura EL. Chagas' disease in a Peruvian Inca mummy. Lancet 1992; 339: 128-129.

50. Lima VS, Iñiguez AM, Otsuki K, Ferreira LF, Araujo A, Vicente ACP, Jansen AM. Chagas disease in ancient hunter-gatherer population, Brazil. Emerg Infect Dis 2008; 14: 1001-1002.

51. Guhl F, Jaramillo C, Vallejo GA, Cárdenas-Arroyo F, Aufderheide A. Chagas disease and human migration. Mem Inst Oswaldo Cruz 2000; 95: 553-555.

52. Guhl F, Jaramillo C, Yockteng V, Vallejo GA, Cárdenas-Arroyo F. Trypanosoma cruzi DNA in human mummies. Lancet 1997; 349: 1370.

53. Guhl F, Jaramillo C, Vallejo GA, Yockteng R, Cárdenas-Arroyo F, 
Fornaciari G, Arriaza B, Aufderheide AC. Isolation of Trypanosoma cruzi DNA in 4,000-year-old mummified human tissue from Northern Chile. Am J Phys Anthropol 1999; 108: 401-417.

54. Bastos OM, Araújo A, Ferreira LF, Santoro A, Wincker P, Morel CM. Experimental paleoparasitology: Identification of T. cruzi DNA in desiccated mouse tissue. Paleopath News 1996; 94: 5-8.

55. Sibley LD. Recent origins among ancient parasites. Vet Parasitol 2003; 115: 185-198.

56. Dubey JP. Toxoplasmosis of animals and humans, 2nd ed. Boca Raton, Florida, USA. CRC Press. 2009.

57. Batz M, Hoffman S, Morris JG Jr. Ranking the risk: the 10 pathogen-food combination with the greatest burden on public health. Florida, USA. University of Florida. 2011.

58. Alvarado-Esquivel C, Cruz-Magallenes HM, Esquivel-Cruz R, Estrada-Martínez S, Rivas-González M, Liesenfeld O, MartínezGarcía SA, Ramírez E, Torres-Castorena A, Castañeda A, Dubey JP. Seroepidemiology of Toxoplasma gondii infections in human adults from three rural communities in Durango State, Mexico. J Parasitol 2008; 94: 811-816.

59. Alvarado-Esquivel C, Rojas-Rivera A, Estrada-Martínez S, Sifuentes-Álvarez A, Liesenfeld O, García-López CR, Dubey JP. Seroepidemiology of Toxoplasma gondii infection in a Mennonite com- munity in Durango State, Mexico. J Parasitol 2010; 96: 941-945.

60. Galuzo IG. The epizootiology of toxoplasmosis. In College of Veterinary Medicine. Toxoplasmosis of Animals. 1st ed. Illinois, USA. University of Illinois. 1970, pp 395-413.

61. Dubey JP, Frenkel JK. Toxoplasmosis of rats: a review, with considerations of their value as an animal model and their possible role in epidemiology. Vet Parasitol 1998; 77: 1-32.

62. Marchiondo AA, Duszynski DW, Maupin GO. Prevalence of antibodies to Toxoplasma gondii in wild and domestic animals of New Mexico, Arizona, and Colorado. J Wildlife Dis 1976; 12: 226-232.

63. Smith DD, Frenkel JK. Prevalence of antibodies to Toxoplasma gondii in wild mammals of Missouri and east central Kansas: biologic and ecologic considerations of transmission. J Wildlife Dis 1995; 31: 15-21.

64. Terra MA, Bellow AR, Bastos OM, Amendoeira MR, Coelho JM, Ferreira LF, Araújo A. Detection of Toxoplasma gondii DNA by polymerase chain reaction in experimentally desiccated tissues. Mem Inst Oswaldo Cruz 2004; 99: 185-188.

65. Morrow JJ, Newby J, Piombino-Mascali D, Reinhard KJ. Taphonomic considerations for the analysis of parasites in archaeological materials. Int J Paleopathol 2016; 13: 56-64. 